\title{
Geometric Thickness of Complete Graphs
}

\author{
Michael B. Dillencourt ${ }^{\star}$, David Eppstein ${ }^{\star \star}$, and Daniel S. Hirschberg \\ Information and Computer Science, University of California, \\ Irvine, CA 92697-3425, USA. \\ \{dillenco, eppstein, dan\}@ics.uci.edu
}

\begin{abstract}
We define the geometric thickness of a graph to be the smallest number of layers such that we can draw the graph in the plane with straight-line edges and assign each edge to a layer so that no two edges on the same layer cross. The geometric thickness lies between two previously studied quantities, the (graph-theoretical) thickness and the book thickness. We investigate the geometric thickness of the family of complete graphs, $\left\{K_{n}\right\}$. We show that the geometric thickness of $K_{n}$ lies between $\lceil(n / 5.646)+0.342\rceil$ and $\lceil n / 4\rceil$, and we give exact values of the geometric thickness of $K_{n}$ for $n \leq 12$ and $n \in\{15,16\}$.
\end{abstract}

\section{Introduction}

Suppose we wish to display a nonplanar graph on a color terminal in a way that minimizes the apparent complexity to a user viewing the graph. One possible approach would be to use straight-line edges, color each edge, and require that two intersecting edges have distinct colors. A natural question then arises: for a given graph, what is the minimum number of colors required?

Or suppose we wish to print a circuit onto a circuit board, using uninsulated wires, so that if two wires cross, they must be on different layers, and that we wish to minimize the number of layers required. If we allow each wire to bend arbitrarily, this problem has been studied previously; indeed, it reduces to the graph-theoretical thickness of a graph, defined below. However, suppose that we wish to further reduce the complexity of the layout by restricting the number of bends in each wire. In particular, if we do not allow any bends, then the question becomes: for a given circuit, what is the minimum number of layers required to print the circuit using straight-line wires?

These two problems motivate the subject of this paper, namely the geometric thickness of a graph. We define $\theta_{g}(G)$, the geometric thickness of a graph $G$, to be the smallest value of $k$ such that we can assign planar point locations to the vertices of $G$, represent each edge of $G$ as a line segment, and assign each edge to one of $k$ layers so that no two edges on the same layer cross. This corresponds to the notion of "real linear thickness" introduced by Kainen [10].

A related notion is that of (graph-theoretical) thickness of a graph, $\theta(G)$, which has been studied extensively [1, 5, 6, 7, 9, 11] and has been defined as the

\footnotetext{
* Supported by NSF Grants CDA-9617349 and CCR-9703572.

** Supported by NSF Grant CCR-9258355 and matching funds from Xerox Corp.
} 
minimum number of planar graphs into which a graph can be decomposed. The key difference between geometric thickness and graph-theoretical thickness is that geometric thickness requires that the vertex placements be consistent at all layers and that straight-line edges be used, whereas graph-theoretical thickness imposes no consistency requirement between layers.

Alternatively, the graph-theoretical thickness can be defined as the minimum number of planar layers required to embed a graph such that the vertex placements agree on all layers but the edges can be arbitrary curves [10. The equivalence of the two definitions follows from the observation that, given any planar embedding of a graph, the vertex locations can be reassigned arbitrarily in the plane without altering the topology of the planar embedding provided we are allowed to bend the edges at will [10]. This observation is easily verified by induction, moving one vertex at a time.

The (graph-theoretical) thickness is now known for all complete graphs [1. 2, 3, 12, 13, and is given by the following formula:

$$
\theta\left(K_{n}\right)=\left\{\begin{array}{cl}
1, & 1 \leq n \leq 4 \\
2, & 5 \leq n \leq 8 \\
3, & 9 \leq n \leq 10 \\
\left\lceil\frac{n+2}{6}\right\rceil, & n>10
\end{array}\right.
$$

Another notion related to geometric thickness is the book thickness of a graph $G, b t(G)$, defined as follows [4]. A book with $k$ pages or a $k$-book, is a line $L$ (called the spine) in 3-space together with $k$ distinct half-planes (called pages) having $L$ as their common boundary. A $k$-book embedding of $G$ is an embedding of $G$ in a $k$-book such that each vertex is on the spine, each edge either lies entirely in the spine or is a curve lying in a single page, and no two edges intersect except at their endpoints. The book thickness of $G$ is then the smallest $k$ such that $G$ has a $k$-book embedding.

It is not hard to see that the book thickness of a graph is equivalent to a restricted version of the geometric thickness where the vertices are required to form the vertices of a convex $n$-gon. This is essentially Lemma 2.1, page 321 of [4]. It follows that $\theta(G) \leq \theta_{g}(G) \leq b t(G)$. It is shown in 4] that $b t\left(K_{n}\right)=\lceil n / 2\rceil$.

In this paper, we focus on the geometric thickness of complete graphs. In Section 2 we provide an upper bound, $\theta_{g}\left(K_{n}\right) \leq\lceil n / 4\rceil$. In Section 3 we provide a lower bound. In particular, we show that $\theta_{g}\left(K_{n}\right) \geq\left\lceil\frac{3-\sqrt{7}}{2}(n+1)\right\rceil \geq\left\lceil\frac{n+1}{5.646}\right\rceil$. This follows from a more precise expression which gives a slightly better lower bound for certain values of $n$.

These lower and upper bounds do not match in general. The smallest values for which they do not match are $n \in\{13,14,15\}$. For these values of $n$, the upper bound on $\theta_{g}\left(K_{n}\right)$ from Section 2 is 4 , and the lower bound from Section 3 is 3 . In Section 4 we resolve one of these three cases by showing that $\theta_{g}\left(K_{15}\right)=4$. For $n=16$ the two bounds match again, but they are distinct for all larger $n$. Section 5 contains a table of the lower and upper bounds on $\theta_{g}\left(K_{n}\right)$ established in this paper for $n \leq 100$ and lists a few open problems. 


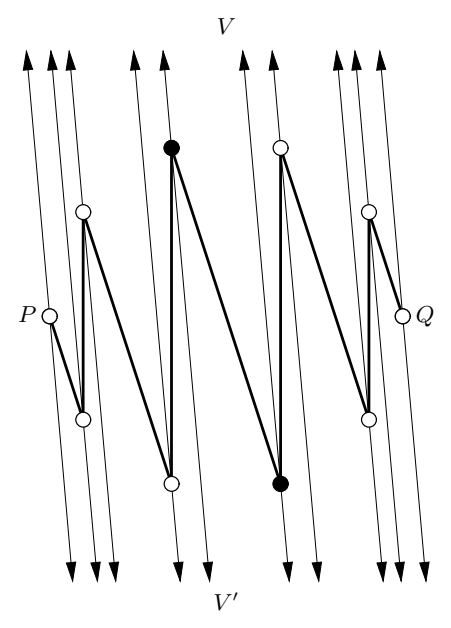

(a)

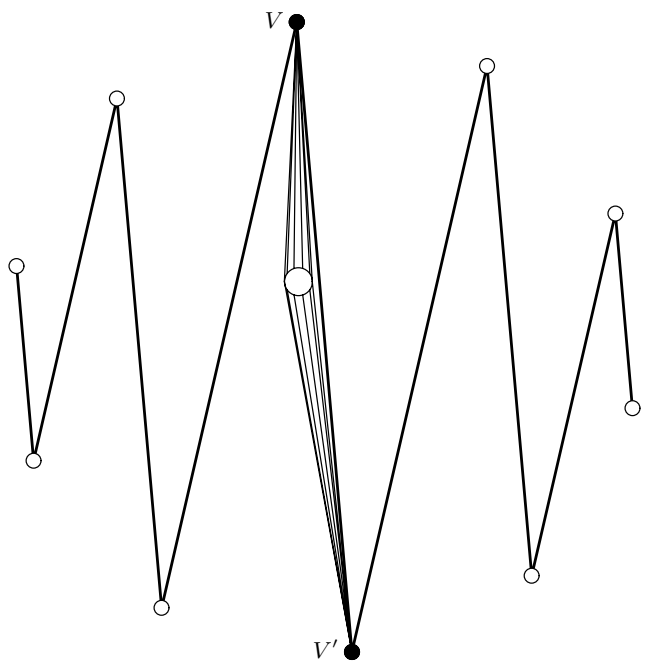

(b)

Fig. 1. Construction for embedding $K_{2 k}$ with geometric thickness of $k / 2$, illustrated for $k=10$. (a) The inner ring. (b) The outer ring. The circle in the center of (b) represents the inner ring shown in (a).

\section{Upper Bounds}

Theorem 1. $\theta_{g}\left(K_{n}\right) \leq\lceil n / 4\rceil$.

Proof. Assume that $n$ is a multiple of 4 , and let $n=2 k$ (so, in particular, $k$ is even). We show that $n$ vertices can be arranged in two rings of $k$ vertices each, an outer ring and an inner ring, so that $K_{n}$ can be embedded using only $k / 2$ layers and with no edges on the same lajer crossing.

The vertices of the inner ring are arranged to form a regular $k$-gon. For each pair of diametrically opposite vertices $P$ and $Q$, consider the zigzag path as illustrated by the thicker lines in Figure 1(a). This path has exactly one diagonal connecting diametrically opposite points (namely, the diagonal connecting the two dark points in the figure.) Note that the union of these zigzag paths, taken over all $k / 2$ pairs of diametrically opposite vertices, contains all $\left(\begin{array}{c}k \\ 2\end{array}\right)$ edges connecting vertices on the inner ring. Note also that for each choice of diametrically opposite vertices, parallel rays can be drawn through each vertex, in two opposite directions, so that none of the rays crosses any edge of the zigzag path. These rays are also illustrated in Figure 1(a).

By continuity, if the infinite endpoints of a collection of parallel rays (e.g., the family of rays pointing "upwards" in Figure प(a)) are replaced by a suitably chosen common endpoint (so that the rays become segments), the common endpoint can be chosen so that none of the segments cross any of the edges of 
the zigzag path. We do this for each collection of parallel rays, thus forming an outer ring of $k$ vertices. This can be done in such a way that the vertices on the outer ring also form a regular $k$-gon. By further stretching the outer ring if necessary, and by moving the inner ring slightly, the figure can be perturbed so that none of the diagonals of the polygon comprising the outer ring intersect the polygon comprising the inner ring. The outer ring constructed in this fashion is illustrated in Figure 1(b).

Once the $2 k$ vertices have been placed as described above, the edges of the complete graph can be decomposed into $k / 2$ layers. Each layer consists of:

1. A zigzag path through the outer ring, as shown in Figure 1(b).

2. All edges connecting $V$ and $V^{\prime}$ to vertices of the inner ring, where $V$ and $V^{\prime}$ are the (unique) pair of diametrically opposite points joined by an edge in the zigzag path through the outer ring. (These edges are shown as edges connecting the circle with $V$ and $V^{\prime}$ in Figure 1 $\mathrm{b}$ ), and as arrows in Figure 1(a)).

3. The zigzag path through the inner ring that does not intersect any of the edges connecting $V$ and $V^{\prime}$ with inner-ring vertices. (These are the heavier lines in Figure 1(a).)

It is straightforward to verify that this is indeed a decomposition of the edges of $K_{n}$ into $k / 2=n / 4$ layers.

\section{Lower Bounds}

Theorem 2. For all $n \geq 1$,

$$
\theta_{g}\left(K_{n}\right) \geq \max _{1 \leq x \leq n / 2} \frac{\left(\begin{array}{l}
n \\
2
\end{array}\right)-2\left(\begin{array}{l}
x \\
2
\end{array}\right)-3}{3 n-2 x-7} .
$$

In particular, for $n \geq 12$,

$$
\theta_{g}\left(K_{n}\right) \geq\left\lceil\frac{3-\sqrt{7}}{2} n+0.342\right\rceil \geq\left\lceil\frac{n}{5.646}+0.342\right\rceil
$$

Proof. We first prove a slightly less precise bound, namely

$$
\theta_{g}\left(K_{n}\right) \geq \frac{3-\sqrt{7}}{2} n-O(1)
$$

For graph $G$ and vertex set $X$, let $G[X]$ denote the subgraph of $G$ induced by $X$. Let $S$ be any planar point set, and let $T_{1}, \ldots T_{k}$ be a set of planar triangulations such that every segment connecting two points in $S$ is an edge of at least one of the $T_{i}$. Find two parallel lines that cut $S$ into three subsets $A, B$, and $C$ (with $B$ the middle set), with $|A|=|C|=x$, where $x$ is a value to be chosen later. For any $T_{i}$, the subgraph $T_{i}[A]$ is connected, because any line joining two vertices of $A$ can be retracted onto a path through $T_{i}[A]$ by moving it away from the 
line separating $A$ from $B$. Similarly, $T_{i}[C]$ is connected, and hence each of the subgraphs $T_{i}[A]$ and $T_{i}[C]$ has at least $x-1$ edges.

By Euler's formula, each $T_{i}$ has at most $3 n-6$ edges, so the number of edges of $T_{i}$ not belonging to $T_{i}[A] \cup T_{i}[C]$ is at most $3 n-6-2(x-1)=3 n-2 x-4$. Hence

$$
\left(\begin{array}{l}
n \\
2
\end{array}\right) \leq 2\left(\begin{array}{l}
x \\
2
\end{array}\right)+k(3 n-2 x-4) .
$$

Solving for $k$, we have

$$
k \geq \frac{\left(\begin{array}{l}
n \\
2
\end{array}\right)-2\left(\begin{array}{l}
x \\
2
\end{array}\right)}{3 n-2 x-4},
$$

and hence

$$
k \geq \frac{n^{2}-2 x^{2}}{6 n-4 x}-O(1) .
$$

If $x=c n$ for some constant $c$, then the fraction in (3.5) is of the form $n(1-$ $\left.2 c^{2}\right) /(6-4 c)$. This is maximized when $c=(3-\sqrt{7}) / 2$. Substituting the value $x=(3-\sqrt{7}) n / 2$ into (3.5) yields (3.3).

To obtain the sharper conclusion of the theorem, observe that by choosing the direction of the two parallel lines appropriately, we can force at least one point of the convex hull of $S$ to lie in $B$. Hence, of the edges of $T_{i}$ that do not belong to $T_{i}[A] \cup T_{i}[C]$, at least three are on the convex hull. If we do not count these three edges, then each $T_{i}$ has at most $3 n-2 x-7$ edges not belonging to $T_{i}[A] \cup T_{i}[C]$, and we can strengthen (3.4) to

$$
\left(\begin{array}{l}
n \\
2
\end{array}\right)-3 \leq 2\left(\begin{array}{l}
x \\
2
\end{array}\right)+k(3 n-2 x-7)
$$

or

$$
k \geq \frac{\left(\begin{array}{l}
n \\
2
\end{array}\right)-2\left(\begin{array}{l}
x \\
2
\end{array}\right)-3}{3 n-2 x-7} .
$$

Since (3.6) holds for any $x$, 3.1) follows.

To prove (3.2), let $f(x)$ be the expression on the right-hand side of (3.6). Consider the inequality $f(x) \geq x_{0}$, where $x_{0}$ is a constant to be specified later. After cross-multiplication, this inequality becomes

$$
-x^{2}+x+\frac{n^{2}}{2}-\frac{n}{2}-3-(3 n-7-2 x) x_{0} \geq 0 .
$$

The expression in the left-hand side of (3.7) represents an inverted parabola in $x$. If we let $x=x_{0}$, we obtain

$$
x_{0}^{2}+(8-3 n) x_{0}+\frac{n^{2}}{2}-\frac{n}{2}-3 \geq 0,
$$

and if we let $x=x_{0}+1$ we obtain the same inequality. Now, consider $x_{0}$ of the form $A n+B-\epsilon$. Choose $A$ and $B$ so that if $\epsilon=0$, the terms involving $n^{2}$ and 
$n$ vanish in (3.8). This gives the values $A=(3-\sqrt{7}) / 2$ and $B=\sqrt{7}(23 / 14)-4$. Substituting $x_{0}=A n+B-\epsilon$ with these values of $A$ and $B$ into (3.8), we obtain

$$
\sqrt{7} \cdot \epsilon \cdot n+\left(\epsilon^{2}-\frac{23 \epsilon}{\sqrt{7}}-3 / 28\right) \geq 0 .
$$

For $\epsilon=0.0045$, (3.9) will be true when $n \geq 12$. Therefore, for all $x \in\left[x_{0}, x_{0}+1\right]$, $f(x) \geq x_{0}$, when $\epsilon=0.0045$ and $n \geq 12$. In particular, $f\left(\left\lceil x_{0}\right\rceil\right) \geq x_{0}$. Since $k$ is an integer, (3.2) follows from (3.6).

\section{The Geometric Thickness of $K_{15}$}

The lower bounds on geometric thickness provided by equation (3.1) of Theorem 2 are asymptotically larger than the lower bounds on graph-theoretical thickness provided by equation (1.1), and they are in fact at least as large for all values of $n \geq 12$. However, they are not tight. In particular, we show that $\theta_{g}\left(K_{15}\right)=4$, even though 3.1 only gives a lower bound of 3 .

Theorem 3. $\theta_{g}\left(K_{15}\right)=4$.

To prove this theorem, we first note that the upper bound, $\theta_{g}\left(K_{15}\right) \leq 4$, follows immediately from Theorem 1 .

To prove the lower bound, assume that we are given a planar point set $S$, with $|S|=15$. We show that there cannot exist a set of three triangulations of $S$ that cover all $\left(\begin{array}{c}15 \\ 2\end{array}\right)=105$ line segments joining pairs of points in $S$. We use the following two facts: (1) A planar triangulation with $n$ vertices and $b$ convex hull vertices contains $3 n-3-b$ edges; and (2) Any planar triangulation of a given point set necessarily contains all convex hull edges. There are several cases, depending on how many points of $S$ lie on the convex hull.

Case 1: 3 points on convex hull. Let the convex hull points be $A, B$ and $C$. Let

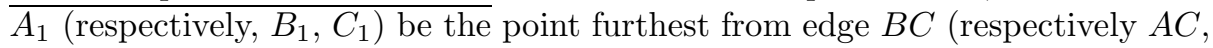
$A B$ ) within triangle $A B C$. Let $A_{2}$ (respectively, $B_{2}, C_{2}$ ) be the point next furthest from edge $B C$ (respectively $A C, A B$ ) within triangle $A B C$.

Lemma 1. The edge $A A_{1}$ will appear in every triangulation of $S$.

Proof. Orient triangle $A B C$ so that edge $B C$ is on the $x$-axis and point $A$ is above the $x$-axis. For an edge $P Q$ to intersect $A A_{1}$, at least one of $P$ or $Q$ must lie above the line parallel to $B C$ that passes through $A_{1}$. But there is only one such point, namely $A$.

Lemma 2. At least one of the edges $A_{1} A_{2}$ or $A A_{2}$ will appear in every triangulation of $S$.

Proof. Orient triangle $A B C$ so that edge $B C$ is on the $x$-axis and point $A$ is above the $x$-axis. For an edge $P Q$ to intersect $A_{1} A_{2}$ or $A A_{2}$, at least one of $P$ or $Q$ must lie above the line parallel to $B C$ that passes through $A_{2}$. There are only 
two such points, $A$ and $A_{1}$. Hence an edge intersecting $A_{1} A_{2}$ must necessarily be $A X$ and an edge intersecting $A A_{2}$ must necessarily be $A_{1} Y$, for some points $X$ and $Y$ outside triangle $A A_{1} A_{2}$. Since edges $A X$ and $A_{1} Y$ both split triangle $A A_{1} A_{2}$, they intersect, so both edges cannot be present in a triangulation. It follows that either $A_{1} A_{2}$ or $A A_{2}$ must be present.

Now let $Z$ be the set of 12 edges consisting of the three convex hull edges and the nine edges $p p_{1}, p p_{2}, p_{1} p_{2}$ (where $p \in\{A, B, C\}$ ). Each triangulation of $S$ contains 39 edges, and since any triangulation contains all three convex hull edges, it follows from Lemmas 1 and 2 that at least 9 edges of any triangulation must belong to $Z$. Hence a triangulation contains at most 30 edges not in $Z$. Thus three triangulations can contain at most $30 \cdot 3+12=102$ edges, and hence cannot contain all 105 edges joining pairs of points in $S$.

Case 2: 4 points on convex hull. Let $A, B, C, D$ be the four convex hull vertices. Assume triangle $D A B$ has at least one point of $S$ in its interior (if not, switch $A$ and $C$ ). Let $A_{1}$ be the point inside triangle $D A B$ furthest from the line $D B$. By Lemma 1, the edge $A A_{1}$ must appear in every triangulation of $S$, as must the 4 convex hull edges. Since any triangulation of $S$ has 38 edges, three triangulations can account for at most $3 \cdot 33+5=104$ edges.

Case 3: 5 or more points on convex hull. Let $h$ be the number of points on the convex hull. A triangulation of $S$ will have $42-h$ edges, and all $h$ hull edges must be in each triangulation. So the total number of edges in three triangulations is at most $3(42-2 h)+h=126-5 h$, which is at most 101 for $h \geq 5$.

This completes the proof of Theorem 3 ,

\section{Final Remarks}

In this paper we have defined the geometric thickness, $\theta_{g}$, of a graph, a measure of approximate planarity that we believe is a natural notion. We have established upper bounds and lower bounds on the geometric thickness of complete graphs. Table 1 contains the upper and lower bounds on $\theta_{g}\left(K_{n}\right)$ for $n \leq 100$.

Many open questions remain about geometric thickness. Here we mention several.

1. Find exact values for $\theta_{g}\left(K_{n}\right)$ (i.e., remove the gap between upper and lower bounds in Table 1). In particular, what are the values for $K_{13}$ and $K_{14}$ ?

2. What is the smallest graph $G$ for which $\theta_{g}(G)>\theta(G)$ ? We note that the existence of a graph $G$ such that $\theta_{g}(G)>\theta(G)$ (e.g., $\left.K_{15}\right)$ establishes Conjecture 2.4 of [10].

3. Is it true that $\theta_{g}(G)=O(\theta(G))$ for all graphs $G$ ? It follows from Theorem 1 that this is true for complete graphs.

4. What is the complexity of computing $\theta_{g}(G)$ for a given graph $G$ ? In particular, is it NP-complete? (Computing $\theta(G)$ is known to be NP-complete [11.) 
Table 1. Upper and lower bounds on $\theta_{g}\left(K_{n}\right)$ established in this paper.

\begin{tabular}{|c|r|r|}
\hline$n$ & LB & UB \\
\hline $1-4$ & 1 & 1 \\
$5-8$ & 2 & 2 \\
$9-12$ & 3 & 3 \\
$13-14$ & 3 & 4 \\
$15-16$ & 4 & 4 \\
$17-20$ & 4 & 5 \\
$21-24$ & 5 & 6 \\
$25-26$ & 5 & 7 \\
$27-28$ & 6 & 7 \\
$29-31$ & 6 & 8 \\
32 & 7 & 8 \\
$33-36$ & 7 & 9 \\
37 & 7 & 10 \\
\hline
\end{tabular}

\begin{tabular}{|c|r|r|}
\hline$n$ & $\mathrm{LB}$ & $\mathrm{UB}$ \\
\hline $38-40$ & 8 & 10 \\
$41-43$ & 8 & 11 \\
44 & 9 & 11 \\
$45-48$ & 9 & 12 \\
$49-52$ & 10 & 13 \\
$53-54$ & 10 & 14 \\
$55-56$ & 11 & 14 \\
$57-60$ & 11 & 15 \\
$61-64$ & 12 & 16 \\
65 & 12 & 17 \\
$66-68$ & 13 & 17 \\
$69-71$ & 13 & 18 \\
72 & 14 & 18 \\
\hline
\end{tabular}

\begin{tabular}{|c|c|c|}
\hline$n$ & $\mathrm{LB}$ & $\mathrm{UB}$ \\
\hline $73-76$ & 14 & 19 \\
77 & 14 & 20 \\
$78-80$ & 15 & 20 \\
$81-82$ & 15 & 21 \\
$83-84$ & 16 & 21 \\
$85-88$ & 16 & 22 \\
$89-92$ & 17 & 23 \\
$93-94$ & 17 & 24 \\
$95-96$ & 18 & 24 \\
$97-99$ & 18 & 25 \\
100 & 19 & 25 \\
\hline
\end{tabular}

Note: Upper bounds are from Theorem 1 The lower bounds for $n \geq 12$ are from Theorem 2 with the exception of the lower bound for $n=15$ which is from Theorem 3 . Lower bounds for $n<12$ are from (1.1).

\section{References}

[1] V. B. Alekseev and V. S. Gončakov. The thickness of an arbitrary complete graph. Math USSR Sbornik, 30(2):187-202, 1976.

[2] L. W. Beineke. The decomposition of complete graphs into planar subgraphs. In F. Harary, editor, Graph Theory and Theoretical Physics, chapter 4, pages 139-153. Academic Press, London, UK, 1967.

[3] L. W. Beineke and F. Harary. The thickness of the complete graph. Canadian Journal of Mathematics, 17:850-859, 1965.

[4] F. Bernhart and P. C. Kainen. The book thickness of a graph. Journal of Combinatorial Theory Series B, 27:320-331, 1979.

[5] R. Cimikowski. On heuristics for determining the thickness of a graph. Information Sciences, 85:87-98, 1995.

[6] A. M. Dean, J. P. Hutchinson, and E. R. Scheinerman. On the thickness and arboricity of a graph. Journal of Combinatorial Theory Series B, 52:147-151, 1991.

[7] J. H. Halton. On the thickness of graphs of given degree. Information Sciences, 54:219-238, 1991.

[8] N. Hartsfield and G. Ringel. Pearls in Graph Theory. Academic Press, Boston, MA, 1990.

[9] B. Jackson and G. Ringel. Plane constructions for graphs, networks, and maps: Measurements of planarity. In G. Hammer and Pallaschke D, editors, Selected Topics in Operations Research and Mathematical Economics: Proceedings of the 8th Symposium on Operations Research, pages 315-324, Karlsruhe, West Germany, August 1983. Springer-Verlag Lecture Notes in Economics and Mathematical Systems 226. 
110 Michael B. Dillencourt, David Eppstein, and Daniel S. Hirschberg

[10] P. C. Kainen. Thickness and coarseness of graphs. Abhandlungen aus dem Mathematischen Seminar der Universität Hamburg, 39:88-95, 1973.

[11] A. Mansfield. Determining the thickness of a graph is NP-hard. Mathematical Proceedings of the Cambridge Philosophical Society, 93(9):9-23, 1983.

[12] J. Mayer. Decomposition de $K_{16}$ en trois graphes planaires. Journal of Combinatorial Theory Series B, 13:71, 1972.

[13] J. Vasak. The thickness of the complete graph having $6 m+4$ points. Manuscript. Cited in [8, 9]. 